\title{
Autovalores e autovetores: utilização na classificação de relevância
}

\author{
Eigenvalues and Eigenvectors : Use on Classification of Relevance
}

\author{
Mylena Roehrs ${ }^{1}$, Larissa Melchiors Furlan² e Glauber Rodrigues de Quadros ${ }^{3}$ \\ ${ }^{1}$ Universidade Federal de Santa Maria - Campus Cachoeira do Sul, Cachoeira do Sul, Brasil \\ mylenaroehrs00@hotmail.com \\ ${ }^{2}$ Universidade Federal de Santa Maria - Campus Cachoeira do Sul, Cachoeira do Sul, Brasil \\ larimelfurlan@hotmail.com \\ ${ }^{3}$ Universidade Federal de Santa Maria - Campus Cachoeira do Sul, Cachoeira do Sul, Brasil \\ glauber.quadros@ufsm.br
}

\section{Resumo}

A utilização de autovalores e autovetores, tópico estudado em Álgebra Linear, estende-se a diversas outras áreas, como à engenharia, genética, geografia, economia, etc. Em todas essas áreas existem muitas aplicações. O principal objetivo deste trabalho é estudar e desenvolver algumas destas aplicações de autovalores e autovetores na resolução de problemas. São apresentados métodos de ranqueamento utilizando-se estes conceitos e apresentando-se situações hipotéticas como exemplos. Além disso, são construidos modelos matemáticos utilizando-se Cadeias de Markov.

Palavras-chave: Autovetores; Ranqueamento; Cadeia de markov

\section{Abstract}

The use of eigenvalues and eigenvectors, topic studied in Linear Algebra, extends to several other areas, such as engineering, genetics, geography, economics, etc. In all these areas there are many applications. The main objective of this work is to study and develop some of these applications of eigenvalues and eigenvectors in solving problems. Methods of ranking are presented using these concepts and presenting hypothetical situations as examples. In addition, mathematical models are constructed using Markov Chains.

Keywords: Eigenvectors; Assignment; Markov chain 


\section{Introdução}

A análise da matemática matricial está presente em quase todos os ramos da engenharia moderna e fazendo o uso da definição da mesma pode-se fazer classificações de relevância, como o ranqueamento do Google, e o algoritmo Page Rank. "PageRank (PR) é uma métrica utilizada pelo Google dentro do seu algorítmo para entender a importância que um site, ou página, tem para ele (Google) frente a Internet. Ele foi desenvolvido em 1995 na Universidade de Stanford por Larry Page, daí vem o nome "Page” Rank" (RICOTTA, 2017). A partir do cálculo de autovalores e autovetores de uma matriz, tem-se várias propriedades que podem ser vistas ao longo desse trabalho. Considerando uma matriz quadrada, o escalar real $\lambda$ é um autovalor associado a um autovetor $\mathrm{v} \neq 0$ se $\mathrm{Av}=\lambda \mathrm{v}$ e pode ser calculado através das raízes dos polinômios característicos de A. Autoridade de domínio, melhor escolha e avaliação de competência são exemplos do que esse uso engloba.

Outra definição matemática usada ao longo desse trabalho é a de Cadeias de Markov, um caso particular de processo estocástico. Usando distribuição de probabilidade, é encontrada a predição de estados seguintes de alguma situação, desde que o estado atual seja conhecido, já que depende apenas do mesmo e não da sequência que o precedeu. Como exemplo, tem-se o processo de nascimento e morte, onde existem apenas 3 possibilidades em cada instante: nascer (acrescentar um elemento); morrer (diminuir um elemento); nada (não acrescentar, nem diminuir). Nota-se que a probabilidade de tudo acontecer não depende do tamanho atual da população. Esse parâmetro tem muitas aplicações na estatística do cotidiano, sendo usado para encontrar um estado estável de uma situação, como será visto num exemplo.

Como objetivo principal do trabalho tem-se a aplicação de autovalores e autovetores em áreas diversas, como na engenharia, na genética, geografia, economia, etc. Também será explicado o mecanismo Page Rank e como forma de esclarecer, serão resolvidos exemplos, com a utilização da Teoria de Grafos na solução de problemas do tipo indicação e votação, além de demonstrar o uso das Cadeias de Markov com exemplos simples e práticos. Como justificativa, traz a importância do uso dessa matemática e sua aplicação no dia-a-dia, usando uma metodologia simplificada e de fácil acesso de modo que a organização das ideias seja acessível à várias áreas.

\section{Desenvolvimento Teórico}

\subsection{Ranqueamento: Algoritmo do Google}

Conhecido como Page Rank, o algoritmo utilizado pelo Google para classificar sites, contabiliza a importância de uma página de acordo com os links que "apontam" para ela. Por exemplo, um site recomendado por muitos outros sites que não têm uma boa recomendação, não vai ter uma alta importância. A quantidade não vale se não há qualidade, para um site ter um ótimo posicionamento, ele deve ser recomendado ou "apontado" por muitas páginas que tem um bom posicionamento. 
Figura 1 - Representação gráfica do Page Rank

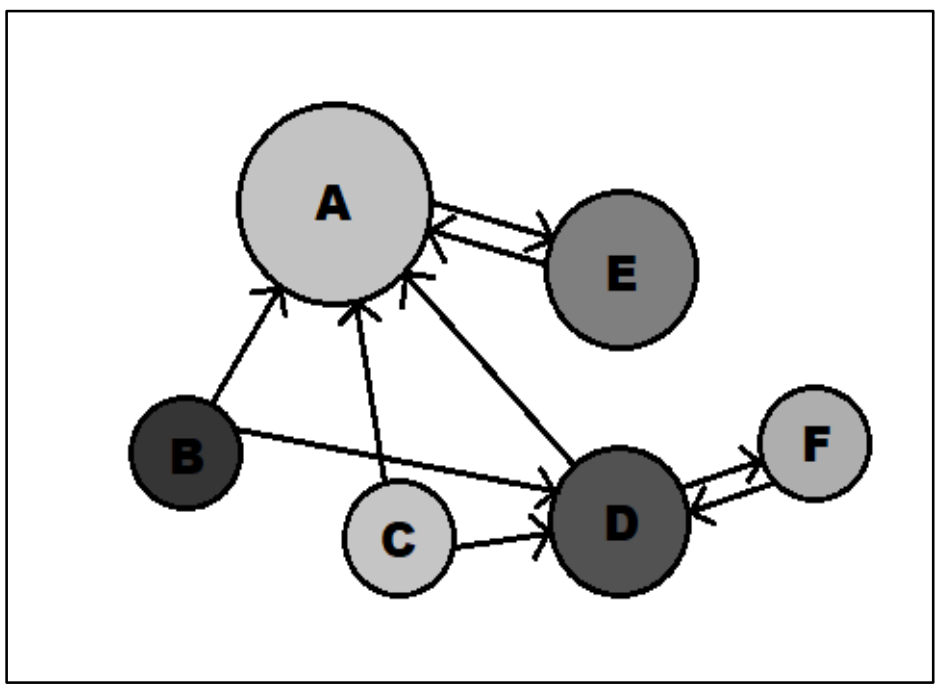

Fonte: próprio autor

\subsubsection{Exemplo do mecanismo}

A matriz que representa a imagem acima é:

$$
\mathrm{T}=\left[\begin{array}{llllll}
\mathbf{0} & \mathbf{1} & \mathbf{1} & \mathbf{1} & \mathbf{1} & \mathbf{0} \\
\mathbf{0} & \mathbf{0} & \mathbf{0} & \mathbf{0} & \mathbf{0} & \mathbf{0} \\
\mathbf{0} & \mathbf{0} & \mathbf{0} & \mathbf{0} & \mathbf{0} & \mathbf{0} \\
\mathbf{0} & \mathbf{1} & \mathbf{1} & \mathbf{0} & \mathbf{0} & \mathbf{1} \\
\mathbf{1} & \mathbf{0} & \mathbf{0} & \mathbf{0} & \mathbf{0} & \mathbf{0} \\
\mathbf{0} & \mathbf{0} & \mathbf{0} & \mathbf{1} & \mathbf{0} & \mathbf{0}
\end{array}\right]
$$

Essa matriz é obtida através da lógica de que, quando há ligação de um elemento e outro, a entrada é 1 e quando o elemento não indica o outro, é 0. Por exemplo, A só se liga em E, por isso a única entrada com 1 na primeira coluna (ligações de $\mathrm{A}$ ), é a entrada correspondente à $\mathrm{T}_{51}$.

Ou seja, considerando um grafo simples de $n$ vértices, a matriz de adjacência é uma matriz $n \times \mathrm{x} n$, onde o valor de cada elemento ejk da matriz é, no caso de grafos orientados em que os vértices vk e vj são ligados por uma aresta no sentido de vk para vj:

$\left\{\begin{array}{l}1, \text { se os vértices } v_{j} \text { e } v_{k} \text { são ligados por uma aresta; } \\ 0 \text {, caso contrário. }\end{array}\right.$

Um vetor $\mathrm{p}=\left(\mathrm{p}_{1}, \mathrm{p}_{2}, \ldots, \mathrm{p}_{\mathrm{n})} \in \mathrm{R}^{\mathrm{n}} \operatorname{com} \mathrm{p}_{\mathrm{i}} \geq 0\right.$ e $\sum_{\boldsymbol{i}=\mathbf{1}}^{\boldsymbol{n}} \boldsymbol{p} \boldsymbol{i}=\mathbf{1}$ é chamado de estocástico e uma matriz $\mathrm{A} \in \mathrm{M}(\mathrm{n}, \mathrm{n})$ cujos vetores coluna são estocásticos é chamada de matriz estocástica. De acordo com o Teorema de PerronFrobenius, caso Markoviano: Seja T uma matriz de transição, nesse caso de adjacência, então

(i) Se $\lambda$ é autovalor de T, então $|\lambda| \leq 1$;

(ii) $\quad \lambda=1$ é autovalor de T. 
Fazendo a transformação para matriz estocástica, obtemos uma matriz onde a soma dos elementos de cada coluna é sempre 1, usando:

$$
P i j=\left\{\begin{array}{l}
a_{i j} / c_{j}, \text { se } c_{j} \neq 0 \\
0, \text { caso contrário }
\end{array}\right.
$$

Com cj $=\sum_{i=1}^{n} \mathbf{a i j}$, temos:

$$
\mathrm{P}=\left[\begin{array}{cccccc}
\mathbf{0} & 1 / 2 & \mathbf{1} / 2 & \mathbf{1} / 2 & \mathbf{1} & \mathbf{0} \\
\mathbf{0} & \mathbf{0} & \mathbf{0} & \mathbf{0} & \mathbf{0} & \mathbf{0} \\
\mathbf{0} & \mathbf{0} & \mathbf{0} & \mathbf{0} & \mathbf{0} & \mathbf{0} \\
\mathbf{0} & \mathbf{1} / 2 & \mathbf{1} / 2 & \mathbf{0} & \mathbf{0} & \mathbf{1} \\
\mathbf{1} & \mathbf{0} & \mathbf{0} & \mathbf{0} & \mathbf{0} & \mathbf{0} \\
\mathbf{0} & \mathbf{0} & \mathbf{0} & \mathbf{1} / 2 & \mathbf{0} & \mathbf{0}
\end{array}\right]
$$

Se tivéssemos ainda uma coluna de zeros, deveríamos usar um vetor d onde as colunas zeradas seriam transformadas em colunas com $1 / \mathrm{n}$, para termos novamente a soma igual a 1 .

Usando essa matriz, temos o autovalor dominante 1, porém com o autovetor relacionado teríamos ciclos ou "becos sem saída" e no fim apenas dois índices teriam relevância, 50\% cada um. Para resolver esse problema, tornamos o grafo interligado irredutível usando um fator de importância $\alpha$, que nesse caso será 0.85 . O valor do fator de importância pode variar, nesse caso sendo empregado um valor comumente usado. Seja J uma matriz nxn cujas entradas são todas iguais a $1 / \mathrm{n}$ e $\mathrm{G}$ a matriz que expressa a resolução do problema citado acima, temos: $\mathrm{G}=\alpha(\mathrm{A})+(1$ $-\alpha) \mathrm{J}$

$$
\mathrm{G}=0.85\left[\begin{array}{cccccc}
0 & 1 / 2 & 1 / 2 & 1 / 2 & 1 & 0 \\
0 & 0 & 0 & 0 & 0 & 0 \\
0 & 0 & 0 & 0 & 0 & 0 \\
0 & 1 / 2 & 1 / 2 & 0 & 0 & 1 \\
1 & 0 & 0 & 0 & 0 & 0 \\
0 & 0 & 0 & 1 / 2 & 0 & 0
\end{array}\right]+0.15\left[\begin{array}{cccccc}
1 / 6 & 1 / 6 & 1 / 6 & 1 / 6 & 1 / 6 & 1 / 6 \\
1 / 6 & 1 / 6 & 1 / 6 & 1 / 6 & 1 / 6 & 1 / 6 \\
1 / 6 & 1 / 6 & 1 / 6 & 1 / 6 & 1 / 6 & 1 / 6 \\
1 / 6 & 1 / 6 & 1 / 6 & 1 / 6 & 1 / 6 & 1 / 6 \\
1 / 6 & 1 / 6 & 1 / 6 & 1 / 6 & 1 / 6 & 1 / 6 \\
1 / 6 & 1 / 6 & 1 / 6 & 1 / 6 & 1 / 6 & 1 / 6
\end{array}\right]
$$

Logo:

$\mathrm{G}=\left[\begin{array}{cccccc}1 / 40 & 9 / 20 & 9 / 20 & 9 / 20 & 7 / 8 & 1 / 40 \\ 1 / 40 & 1 / 40 & 1 / 40 & 1 / 40 & 1 / 40 & 1 / 40 \\ 1 / 40 & 1 / 40 & 1 / 40 & 1 / 40 & 1 / 40 & 1 / 40 \\ 1 / 40 & 9 / 20 & 9 / 20 & 1 / 40 & 1 / 40 & 7 / 8 \\ 7 / 8 & 1 / 40 & 1 / 40 & 1 / 40 & 1 / 40 & 1 / 40 \\ 1 / 40 & 1 / 40 & 1 / 40 & 9 / 20 & 1 / 40 & 1 / 40\end{array}\right]$

O autovalor dominante da matriz é 1 e o autovetor correspondente é v $=(579 / 100,7 / 20,7 / 20,151 / 100,53 / 10$, 1). O autovetor correspondente é divido pela soma e o resultado dessa divisão para cada componente é a sua porcentagem.

$\operatorname{SOMA}=579 / 100+7 / 20+7 / 20+151 / 100+53 / 10+1=\|143 / 10\|$ 
Então, aproximadamente, temos: $\mathrm{A}=40 \% ; \mathrm{B}=4 \% ; \mathrm{C}=4 \% ; \mathrm{D}=10 \% ; \mathrm{E}=36 \% ; \mathrm{F}=6 \%$;

Como podemos ver acima, a dependência de um elemento ser relevante se dá a partir de quantos outros elementos ele indica, se "liga", ou por quantos é indicado. Conforme um elemento indica outro, a relevância do mesmo é dividida entre ele e os que foram indicados por ele, ou seja, se for indicado por vários elementos que receberam indicações, ele vai ter um pouco de cada uma dessas indicações, fazendo sua importância aumentar. No exemplo anterior, o elemento A é bem indicado e tem uma boa porcentagem. Já E, que é indicado apenas por A, também tem uma boa porcentagem. Isso se dá pelo simples fato de que A transfere boa parte da sua relevância para E, que devolve sua relevância criando um ciclo que faz ambos terem uma boa porcentagem. Mas e se E não indicasse A ?

Nesse caso, temos o seguinte grafo:

Figura 2 - Representação do Page Rank

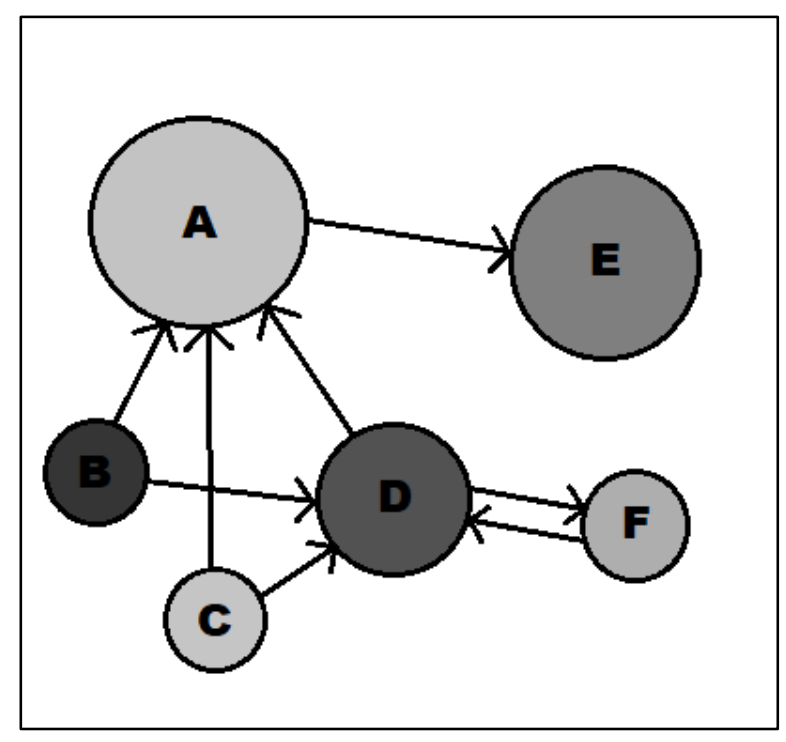

Fonte: próprio autor

A matriz que o representa é:

$$
\left[\begin{array}{llllll}
\mathbf{0} & \mathbf{1} & \mathbf{1} & \mathbf{1} & \mathbf{1} & \mathbf{0} \\
\mathbf{0} & \mathbf{0} & \mathbf{0} & \mathbf{0} & \mathbf{0} & \mathbf{0} \\
\mathbf{0} & \mathbf{0} & \mathbf{0} & \mathbf{0} & \mathbf{0} & \mathbf{0} \\
\mathbf{0} & \mathbf{1} & \mathbf{1} & \mathbf{0} & \mathbf{0} & \mathbf{1} \\
\mathbf{0} & \mathbf{0} & \mathbf{0} & \mathbf{0} & \mathbf{0} & \mathbf{0} \\
\mathbf{0} & \mathbf{0} & \mathbf{0} & \mathbf{1} & \mathbf{0} & \mathbf{0}
\end{array}\right]
$$

E a estocástica obtida:

$$
\left[\begin{array}{cccccc}
1 / 6 & 1 / 2 & 1 / 2 & 1 / 2 & 1 & 0 \\
1 / 6 & 0 & 0 & 0 & 0 & 0 \\
1 / 6 & 0 & 0 & 0 & 0 & 0 \\
1 / 6 & 1 / 2 & 1 / 2 & 0 & 0 & 1 \\
1 / 6 & 0 & 0 & 0 & 0 & 0 \\
1 / 6 & 0 & 0 & 1 / 2 & 0 & 0
\end{array}\right]
$$


Nota-se que aqui usamos o vetor $d$ para que as colunas zeradas se transformassem em colunas com $1 / \mathrm{n}$, obtendo a soma igual a 1 .

Usando o fator de importância $\alpha=0.85$ obtemos a matriz do grafo irredutível:

$$
\mathrm{G}=0.85\left[\begin{array}{cccccc}
1 / 6 & 1 / 2 & 1 / 2 & 1 / 2 & 1 & 0 \\
1 / 6 & 0 & 0 & 0 & 0 & 0 \\
1 / 6 & 0 & 0 & 0 & 0 & 0 \\
1 / 6 & 1 / 2 & 1 / 2 & 0 & 0 & 1 \\
1 / 6 & 0 & 0 & 0 & 0 & 0 \\
1 / 6 & 0 & 0 & 1 / 2 & 0 & 0
\end{array}\right]+0.15\left[\begin{array}{cccccc}
1 / 6 & 1 / 6 & 1 / 6 & 1 / 6 & 1 / 6 & 1 / 6 \\
1 / 6 & 1 / 6 & 1 / 6 & 1 / 6 & 1 / 6 & 1 / 6 \\
1 / 6 & 1 / 6 & 1 / 6 & 1 / 6 & 1 / 6 & 1 / 6 \\
1 / 6 & 1 / 6 & 1 / 6 & 1 / 6 & 1 / 6 & 1 / 6 \\
1 / 6 & 1 / 6 & 1 / 6 & 1 / 6 & 1 / 6 & 1 / 6 \\
1 / 6 & 1 / 6 & 1 / 6 & 1 / 6 & 1 / 6 & 1 / 6
\end{array}\right]
$$

Temos:

$$
\mathrm{G}=\left[\begin{array}{cccccc}
1 / 6 & 9 / 20 & 9 / 20 & 9 / 20 & 7 / 8 & 1 / 40 \\
1 / 6 & 1 / 40 & 1 / 40 & 1 / 40 & 1 / 40 & 1 / 40 \\
1 / 6 & 1 / 40 & 1 / 40 & 1 / 40 & 1 / 40 & 1 / 40 \\
1 / 6 & 9 / 20 & 9 / 20 & 1 / 40 & 1 / 40 & 7 / 8 \\
1 / 6 & 1 / 40 & 1 / 40 & 1 / 40 & 1 / 40 & 1 / 40 \\
1 / 6 & 1 / 40 & 1 / 40 & 9 / 20 & 1 / 40 & 1 / 40
\end{array}\right]
$$

O autovalor dominante é 1 e o autovetor correspondente é $\mathrm{v}=(81 / 50,9 / 25,9 / 25,38 / 25,9 / 25,1)$. A soma é $523 / 100$ e as porcentagens nesse caso são:

$$
\mathrm{A}=31 \% ; \mathrm{B}=7 \% ; \mathrm{C}=7 \% ; \mathrm{D}=29 \% ; \mathrm{E}=7 \% ; \mathrm{F}=19 \% \text {; }
$$

A imagem que representa a matriz e essas porcentagens relacionadas é:

Figura 3 - Representação do Page Rank

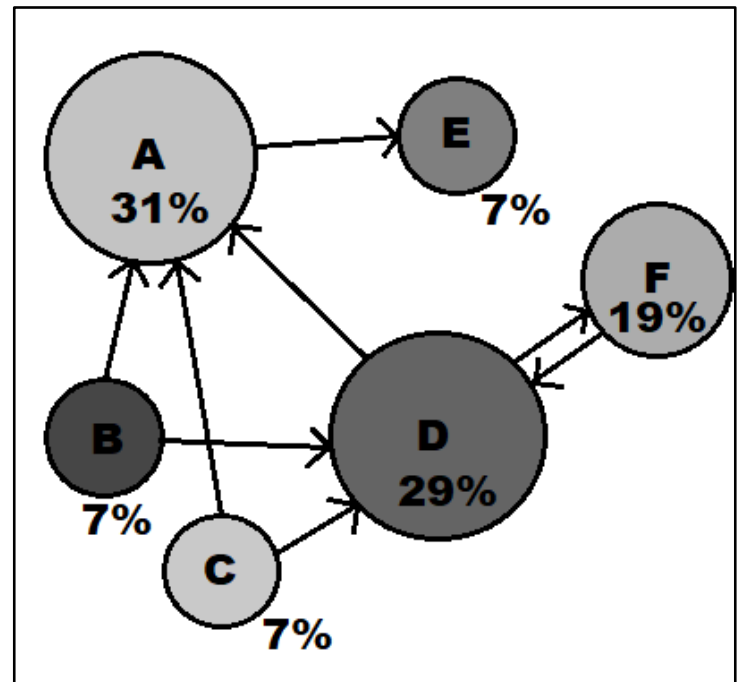

Fonte: próprio autor

Observe que, quando E não indica $\mathrm{A}$, a relevância de ambos diminui, permitindo que os demais tenham sua importância aumentada. A continua tendo a maior porcentagem mas note como E, que não indica ninguém, tem uma baixa, ainda vinda de A. D aumentou sem o ciclo AE, o que fez F aumentar também e ter um ciclo DF, que mantem 
ambos com boa relevância. Quando um elemento deixa de "ajudar" seu grupo, constituindo um ciclo, por exemplo, ele diminui as relevâncias ligadas aquele grupo.

\subsection{Exemplo utilizando o mesmo método}

Determine o ranking para 6 empresas de logística que formam um grupo de indicações entre si. Sendo que:

(i) A empresa 1 indica a empresa 4;

(ii) A empresa 2 indica apenas a empresa 1;

(iii) A empresa 3 indica a empresa 2 e a empresa 4;

(iv) A empresa 4 indica a empresa 1, a 2 e a 3;

(v) A empresa 5 indica apenas a empresa 2.

O grafo que representa os dados é:

Figura 4 - Representação do grafo

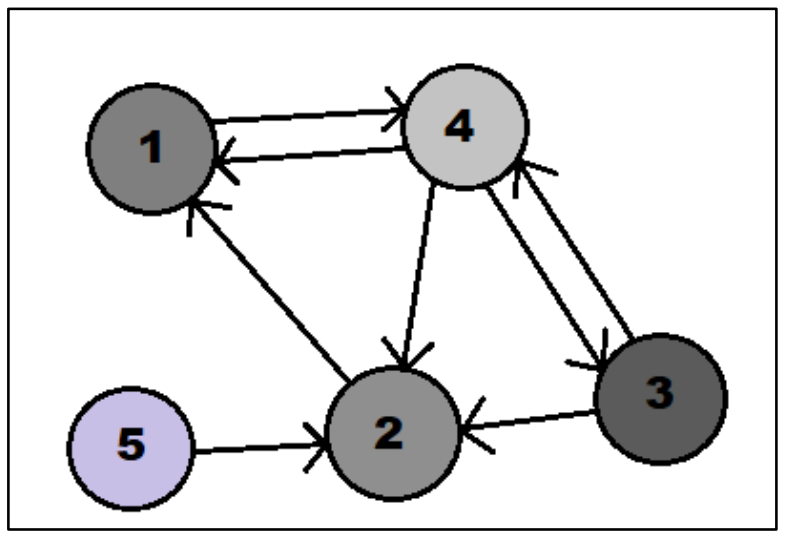

Fonte: próprio autor

Usando a mesma lógica do Page Rank e suas indicações, a matriz correspondente é:

$$
\left[\begin{array}{lllll}
\mathbf{0} & \mathbf{1} & \mathbf{0} & \mathbf{1} & \mathbf{0} \\
\mathbf{0} & \mathbf{0} & \mathbf{1} & \mathbf{1} & \mathbf{1} \\
\mathbf{0} & \mathbf{0} & \mathbf{0} & \mathbf{1} & \mathbf{0} \\
\mathbf{1} & \mathbf{0} & \mathbf{1} & \mathbf{0} & \mathbf{0} \\
\mathbf{0} & \mathbf{0} & \mathbf{0} & \mathbf{0} & \mathbf{0}
\end{array}\right]
$$

Primeiro, obtemos a matriz estocástica através do método já usado:

$$
\left[\begin{array}{ccccc}
\mathbf{0} & \mathbf{1} & \mathbf{0} & \mathbf{1} / 3 & \mathbf{0} \\
\mathbf{0} & \mathbf{0} & \mathbf{1} / 2 & \mathbf{1} / 3 & \mathbf{1} \\
\mathbf{0} & \mathbf{0} & \mathbf{0} & \mathbf{1} / 3 & \mathbf{0} \\
\mathbf{1} & \mathbf{0} & \mathbf{1} / 2 & \mathbf{0} & \mathbf{0} \\
\mathbf{0} & \mathbf{0} & \mathbf{0} & \mathbf{0} & \mathbf{0}
\end{array}\right]
$$


Usando o fator de importância $\alpha=0.85$, tonamos o grafo irredutível e assim corrigindo esse problema. Tendo a matriz $\mathrm{J}$ como uma matriz com todas as entradas iguais a $1 / 5$, temos:

$$
\begin{aligned}
& \mathrm{G}=\alpha(\mathrm{A})+(1-\alpha) \mathrm{J} \\
& \mathrm{G}=0.85\left[\begin{array}{ccccc}
0 & 1 & 0 & 1 / 3 & 0 \\
0 & 0 & 1 / 2 & 1 / 3 & 1 \\
0 & 0 & 0 & 1 / 3 & 0 \\
1 & 0 & 1 / 2 & 0 & 0 \\
0 & 0 & 0 & 0 & 0
\end{array}\right]+0.15\left[\begin{array}{ccccc}
1 / 5 & 1 / 5 & 1 / 5 & 1 / 5 & 1 / 5 \\
1 / 5 & 1 / 5 & 1 / 5 & 1 / 5 & 1 / 5 \\
1 / 5 & 1 / 5 & 1 / 5 & 1 / 5 & 1 / 5 \\
1 / 5 & 1 / 5 & 1 / 5 & 1 / 5 & 1 / 5 \\
1 / 5 & 1 / 5 & 1 / 5 & 1 / 5 & 1 / 5
\end{array}\right]
\end{aligned}
$$

Logo:

$\left[\begin{array}{cccll}3 / 100 & 22 / 25 & 3 / 100 & 47 / 150 & 3 / 100 \\ 3 / 100 & 3 / 100 & 91 / 200 & 47 / 150 & 22 / 25 \\ 3 / 100 & 3 / 100 & 3 / 100 & 47 / 150 & 3 / 100 \\ 22 / 25 & 3 / 100 & 91 / 200 & 3 / 100 & 3 / 100 \\ 3 / 100 & 3 / 100 & 3 / 100 & 3 / 100 & 3 / 100\end{array}\right]$

O maior autovalor da matriz é 1 e seu autovetor associado é v $=(100083 / 10000,683403 / 100000$, 104983/25000, 112917/10000,1). Dividimos cada componente pela soma total das componentes para obter a porcentagem de relevância de cada empresa para fazer seu ranking.

Obtemos: $1=30 \% ; 2=20 \% ; 3=12 \% ; 4=35 \% ; 5=3 \%$.

O ranking de empresas mais bem indicadas no grupo das 6 empresas informadas, portanto, é: $1^{\circ}$ empresa: 4; $2^{\circ}$ empresa: $1 ; 3^{\circ}$ empresa: $2 ; 4^{\circ}$ empresa: $3 ; 5^{\circ}$ empresa: 5 .

Observamos que a melhor empresa é a 4, que é bem indicada, indica outras e por isso faz parte de ciclos que a mantém com uma boa importância.

\subsection{Cadeia de Markov}

"Processo no qual a probabilidade de o sistema estar em um certo estado depende somente de seu estado precedente". (KOLMAN; HILL, 2014, p. 427)

A matriz do sistema é feita a partir dos dados de probabilidade do mesmo; probabilidade do sistema estar no estado $j$ em um período e em um estado $i$ em um período seguinte. Monta-se a matriz de Markov arranjando esses dados conforme seus estados (linhas e colunas).

Após ter a matriz associada, ela é multiplicada pelo seu VETOR DE ESTADO INICIAL, que é um vetor em matriz que suporta os dados iniciais do sistema; O primeiro resultado seria o vetor de estado no dia 1, por exemplo, sendo multiplicado novamente pela matriz e assim por diante, até achar ou não um estado estável. 


\subsubsection{Exemplo usando a cadeia}

Em uma cidade, os habitantes tem quatro opções de pratos principais em um restaurante bom e barato: ravióli de queijo, espaguete com tomate, costela assada e lasanha vegetariana; Suponha que:

- Dos que escolheram ravióli, 35\% escolhem espaguete, 28\% escolhem lasanha e 7\% escolhem costela na próxima noite;

- Dos que escolheram espaguete, 32\% escolhem costela, 18\% escolhem lasanha e 30\% escolhem ravióli na próxima noite;

- Dos que escolheram lasanha, 20\% escolhem ravióli, 25\% escolhem espaguete e 5\% escolhem costela na próxima noite;

- Dos que escolheram costela, 4\% escolhem lasanha, 15\% escolhem ravióli e 15\% escolhem espaguete na próxima noite.

Se em uma noite o público é dividido igualmente, qual a proporção de pratos escolhidos depois de duas noites? E depois de muito tempo?

Resolução do problema:

Usando $(0.25,0.25,0.25,0.25)$ como o vetor de estado inicial, temos:

$\left[\begin{array}{llll}\mathbf{0}, 30 & \mathbf{0 , 3 0} & \mathbf{0 , 2 0} & \mathbf{0 , 1 5} \\ \mathbf{0 , 3 5} & \mathbf{0 , 2 0} & \mathbf{0 , 2 5} & \mathbf{0 , 1 5} \\ \mathbf{0 , 2 8} & \mathbf{0 , 1 8} & \mathbf{0 , 5 0} & \mathbf{0 , 0 4} \\ \mathbf{0 , 0 7} & \mathbf{0 , 3 2} & \mathbf{0 , 0 5} & \mathbf{0 , 6 6}\end{array}\right] \cdot\left[\begin{array}{l}\mathbf{0 , 2 5} \\ \mathbf{0 , 2 5} \\ \mathbf{0 , 2 5} \\ \mathbf{0 , 2 5}\end{array}\right]=$

$\left[\begin{array}{c}0,2375 \\ 0.2375 \\ 0.25 \\ 0.275\end{array}\right]$ (Vetor do estado na primeira noite).

$\left[\begin{array}{llll}0,30 & 0,30 & 0,20 & 0,15 \\ 0,35 & 0,20 & 0,25 & 0,15 \\ 0,28 & 0,18 & 0,50 & 0,04 \\ 0,07 & 0,32 & 0,05 & 0,66\end{array}\right] \cdot\left[\begin{array}{c}0,2375 \\ 0.2375 \\ 0.25 \\ 0.275\end{array}\right]=$

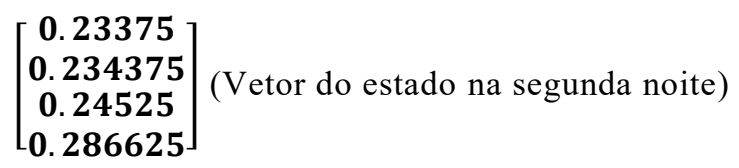


Fazendo essa multiplicação até encontrar o equilíbrio, obtemos:

$\left[\begin{array}{l}\mathbf{0 . 2 3} \\ \mathbf{0 . 2 3} \\ \mathbf{0 . 2 4} \\ \mathbf{0 . 3 0}\end{array}\right]$ (Vetor aproximado do estado em equilíbrio)

Portanto, a proporção de pedidos depois de duas semanas é:

(i) Ravióli de queijo: 23\%

(ii) Espaguete com tomate: $23 \%$

(iii) Lasanha vegetariana: $25 \%$

(iv) Costela assada: $29 \%$

Proporção de pedidos depois de muito tempo:

(i) Ravióli de queijo: 23\%

(ii) Espaguete com tomate: $23 \%$

(iii) Lasanha vegetariana: $24 \%$

(iv) Costela assada: $30 \%$

As porcentagens têm pouca variação devido aos valores iniciais não diferirem muito.

\subsection{Exemplo de classificação por relevância}

Uma forma diferente de exemplificar a classificação por relevância é usar o método anterior em uma situação envolvendo uma eleição e grupos de pessoas, partindo de votos entre essas pessoas.

Há, neste caso, uma votação acontecendo para decidir o presidente de uma ONG. O presidente anterior desistiu do cargo logo antes de ter que tomar uma grande decisão pela organização. Devido a isso, a votação para um novo representante que escolherá entre duas opções de decisão fez-se necessária. As seis pessoas que concorrem ao cargo são as mesmas seis pessoas votantes, dentre elas três apoiam a primeira opção e outras três apoiam a segunda opção de decisão. Dentro desse esquema, cada pessoa deve indicar até duas das seis envolvidas e o esperado é que essas indicações aconteçam dentro de seu grupo de opinião. Porém, após pensar a respeito da decisão que irá ser tomada logo após a eleição, o candidato quatro decide votar em um dos candidatos do outro grupo. O grafo que representa os votos finais da eleição é o seguinte: 
Figura 5 - Representação do grafo

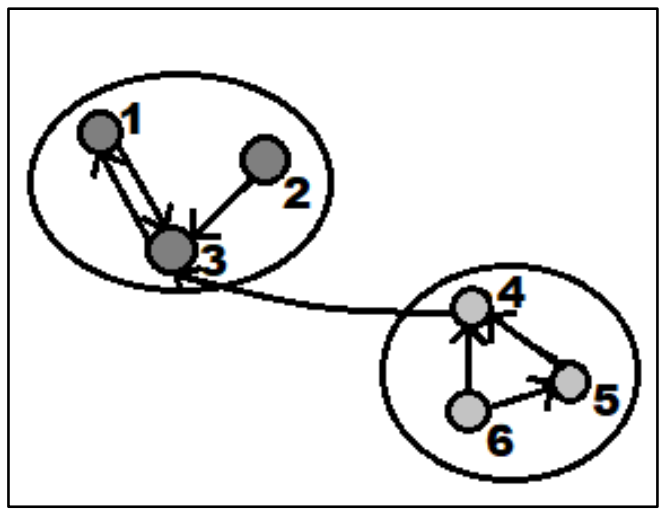

Fonte: próprio autor

Note a ligação da pessoa número 4 com o grupo 1. De que forma essa ligação não esperada afetará no resultado?

A matriz que representa o grafo é:

$$
\left[\begin{array}{llllll}
\mathbf{0} & \mathbf{0} & \mathbf{1} & \mathbf{0} & \mathbf{0} & \mathbf{0} \\
\mathbf{0} & \mathbf{0} & \mathbf{0} & \mathbf{0} & \mathbf{0} & \mathbf{0} \\
\mathbf{1} & \mathbf{1} & \mathbf{0} & \mathbf{1} & \mathbf{0} & \mathbf{0} \\
\mathbf{0} & \mathbf{0} & \mathbf{0} & \mathbf{0} & \mathbf{1} & \mathbf{1} \\
\mathbf{0} & \mathbf{0} & \mathbf{0} & \mathbf{0} & \mathbf{0} & \mathbf{1} \\
\mathbf{0} & \mathbf{0} & \mathbf{0} & \mathbf{0} & \mathbf{0} & \mathbf{0}
\end{array}\right]
$$

Utilizando o mesmo passo a passo já usado nos problemas anteriores, obtemos a matriz estocástica para termos a soma das colunas igual a 1. Dessa forma, temos:

$$
\left[\begin{array}{llllll}
\mathbf{0} & \mathbf{0} & \mathbf{1} & \mathbf{0} & \mathbf{0} & \mathbf{0} \\
\mathbf{0} & \mathbf{0} & \mathbf{0} & \mathbf{0} & \mathbf{0} & \mathbf{0} \\
\mathbf{1} & \mathbf{1} & \mathbf{0} & \mathbf{1} & \mathbf{0} & \mathbf{0} \\
\mathbf{0} & \mathbf{0} & \mathbf{0} & \mathbf{0} & \mathbf{1} & \mathbf{1} / 2 \\
\mathbf{0} & \mathbf{0} & \mathbf{0} & \mathbf{0} & \mathbf{0} & \mathbf{1} / 2 \\
\mathbf{0} & \mathbf{0} & \mathbf{0} & \mathbf{0} & \mathbf{0} & \mathbf{0}
\end{array}\right]
$$

Como temos novamente o problema com os ciclos, usamos o método do fator de importância com $\alpha=0.85$ :

$$
\mathrm{G}=0.85\left[\begin{array}{llllll}
0 & 0 & 1 & 0 & 0 & 0 \\
0 & 0 & 0 & 0 & 0 & 0 \\
1 & 1 & 0 & 1 & 0 & 0 \\
0 & 0 & 0 & 0 & 1 & 1 / 2 \\
0 & 0 & 0 & 0 & 0 & 1 / 2 \\
0 & 0 & 0 & 0 & 0 & 0
\end{array}\right]+0.15\left[\begin{array}{cccccc}
1 / 6 & 1 / 6 & 1 / 6 & 1 / 6 & 1 / 6 & 1 / 6 \\
1 / 6 & 1 / 6 & 1 / 6 & 1 / 6 & 1 / 6 & 1 / 6 \\
1 / 6 & 1 / 6 & 1 / 6 & 1 / 6 & 1 / 6 & 1 / 6 \\
1 / 6 & 1 / 6 & 1 / 6 & 1 / 6 & 1 / 6 & 1 / 6 \\
1 / 6 & 1 / 6 & 1 / 6 & 1 / 6 & 1 / 6 & 1 / 6 \\
1 / 6 & 1 / 6 & 1 / 6 & 1 / 6 & 1 / 6 & 1 / 6
\end{array}\right]=
$$




$\left[\begin{array}{cccccc}1 / 40 & 1 / 40 & 7 / 8 & 1 / 40 & 1 / 40 & 1 / 40 \\ 1 / 40 & 1 / 40 & 1 / 40 & 1 / 40 & 1 / 40 & 1 / 40 \\ 7 / 8 & 7 / 8 & 1 / 40 & 7 / 8 & 1 / 40 & 1 / 40 \\ 1 / 40 & 1 / 40 & 1 / 40 & 1 / 40 & 1 / 40 & 9 / 20 \\ 1 / 40 & 1 / 40 & 1 / 40 & 1 / 40 & 7 / 8 & 9 / 20 \\ 1 / 40 & 1 / 40 & 1 / 40 & 1 / 40 & 1 / 40 & 1 / 40\end{array}\right]$

Como esperado, obtemos o autovalor igual a 1 da matriz com o autovetor relacionado $\mathrm{v}=(12.9804,1$, $14.0946,1.425,9.5,1)$. Somamos todas as componentes e dividimos cada uma delas por essa soma total para obter a porcentagem de voto de cada candidato. Com isso, obtemos:

Candidato 1: $32 \%$; candidato 2: $2.5 \%$; candidato 3: $35 \%$; candidato 4: $4 \%$; candidato 5: $24 \%$; candidato 6 : $2.5 \%$

Como podemos ver, o candidato número 3 é o mais bem votado com $35 \%$ dos votos. Os candidatos 1 e 3 , que possuem boa relevância, também votam entre si, aumentando a importância da sua ligação. No momento em que o candidato 4, que é votado por duas pessoas, "trai" o próprio grupo e vota em alguém do outro, sua importância cai, pois, o candidato não devolve seu voto. A decisão do candidato 4 talvez tenha sido a responsável pela vitória do candidato 3 e, consequentemente, da primeira opinião sobre a decisão a ser tomada.

\section{Conclusões}

As aplicações matemáticas podem ser muito comuns no cotidiano, tanto as mais simplificadas quanto as mais complexas. Essa matemática matricial, como foi visto ao longo do trabalho, pode ser utilizada para ranquear e classificar. Utilizando o método do mecanismo Page Rank é possível obter a classificação de relevância em diversos casos. Com um bom entendimento sobre grafos, matrizes adjacentes, processos estocásticos e seus métodos e a base da matemática matricial, seus autovalores e autovetores, obtém-se esses resultados a partir de alguns dados.

Esse trabalho buscou o aprofundamento das aplicações de proposições conhecidas em diferentes áreas onde pôde ser usado o ranqueamento e encontrado resultados que ficaram de acordo com teoremas apresentados. A importância do conhecimento e do aprofundamento da execução prática do assunto é visível de muitas formas e, em consequência disso, deixa muitas portas abertas para estudos futuros do mesmo.

\section{Referências}

KOLMAN, B.; HILL, D. Álgebra linear com aplicações 9 ed. Rio de Janeiro: LTC, 2014.

ANTON, H.; RORRES, C. Álgebra linear com aplicações. Porto Alegre: Bookman, 2012.

PAIVA, Afonso. Autovalores e Autovetores. 52 slides. Material apresentado para o Departamento de Matemática aplicada e Estatística da USP - São Carlos.

RESNICK, S. Adventures in Stochastic Processes. Birkh, 1992.

HINOJOSA, A.; MILANÉS, A. Apostila de Introdução aos Processos Estocásticos com Aplicações para o Departamento de Estatística ICEx da UFMG. Belo Horizonte.

LAMBERTI, F. Cadeias de Markov e Aplicações. Trabalho de graduação para o curso de Matemática bacharelado da UFSM - Santa Maria, 2015.

BOLDRINI, J. L. Álgebra Linear. 3 ed. São Paulo: Harbra, 1986. 